Reprod. Nutr. Dévelop., 1982, 22 (3), 537-555.

\title{
Etude de I'ovogenèse chez Centropages typicus (Copepoda, Calanoida)
}

\author{
J. ARNAUD, M. BRUNET, J. MAZZA
}

Laboratoire de Biologie animale (Plancton)

Université de Provence, 3, place Victor-Hugo, 13331 Marseille Cedex 3, France.

Summary. A study of oogenesis in Centropages typicus (Copepoda, Calanoida).

We carried out a complete study of oogenesis in Centropages typicus using structural, ultrastructural and cytochemical data. The usual stages of oogenesis, i.e. germinative phase, premeiosis, primary and secondary vitellogenesis, were found. The latter two stages were the most typical. Primary vitellogenesis consisted of endogenous yolk accumulations ; these substances, probably of lipoprotein or lipoglycoprotein nature, were produced at the granular endoplasmic reticulum level and then stocked in the reticulum cavities. During secondary vitellogenesis, endogenous yolk production continued, but we mainly observed the development of exogenous yolk accumulation (lipid droplets and protein globules) in the ooplasm. These accumulations resulted from the fusion of very numerous pinocytotic vesicles arising from the oolemma and containing substances probably brought to the oocytes by the hemolymph. The effect of various proteases on the vitellus globules caused a more or less marked digestion of their contents, tending to prove their protein nature. The end of vitellogenesis was marked by the appearance of vacuolar formations with dense lamellae which could correspond to cortical granules.

\section{Introduction.}

Au cours des quinze dernières années, l'ovogenèse a été étudiée en détail sur les plans ultrastructural et cytochimique chez plusieurs espèces de Malacostracés. En revanche, chez les Entomostracés les travaux sont beaucoup plus rares. On ne peut guère citer que ceux effectués chez divers Branchiopodes des genres Artemia (Anteunis et al., 1966 a et b), Triops (Trentini et Sabelli Scanabissi, 1978 ; Sabelli Scanabissi et Trentini, 1979) et Daphnia (Kessel, 1968), et encore s'agit-il de recherches portant davantage sur des points particuliers de l'ovogenèse que sur son déroulement complet. En ce qui concerne les Copépodes, il convient de mentionner deux études d'ensemble, réalisées en microscopie optique, chez les Calanoides Calanus finmarchicus (Hilton, 1932) et Epilabidocera amphitrites (Park, 1966) ; Razouls a suivi la différer ciation des gonades au cours des stades post-lavaires (copépodites) et adulte chez deux autres Calanoides, Temora stylifera $(1973,1974,1981)$ et Centropages typicus (1975) ; signalons enfin quelques observations ultrastructurales préliminaires effectuées chez Cala- 
nus finmarchicus (Raymont et al., 1974) et Mytilicola intestinalis (Caligoide parasite de la moule) (Durfort, 1977) ; mais ces travaux ne fournissent aucune indication précise sur le déroulement de la vitellogenèse. Dans le cadre de nos investigations en cours sur la biologie digestive des Copépodes (Arnaud et al., 1978 et 1980), notamment sur la destinée des substances absorbées, nous nous sommes intéressés aux modalités de la vitellogenèse, qui, chez les Malacostracés, comporte une phase exogène importante; mais l'insuffisance des données actuelles sur l'ovogenèse des Copépodes nous a conduits à en étudier la totalité des phases sur les plans structural et ultrastructural ; des tests cytochimiques complètent les résultats obtenus essentiellement chez Centropages typicus Kröyer 1849, qui est l'une des espèces les plus communes dans le golfe de Marseille.

\section{Matériel et méthodes.}

Les spécimens femelles ont été étudiés sur coupes, soit après inclusion à la paraffine selon des méthodes utilisées antérieurement (Arnaud et al., 1978), soit, le plus souvent, selon les techniques suivantes de microscopie électronique.

1. Méthodes de fixation-inclusion.

a) Recherches morphologiques. Fixation : $8-20 \mathrm{~h}$ à $4{ }^{\circ} \mathrm{C}$ dans une solution de cacodylate de $\mathrm{Na}(0,2 \mathrm{M}, \mathrm{pH} 7,2)+$ glutaraldéhyde (2-3 p. 100) + paraformaldéhyde $(1$ p. 100$)+$ eau de mer $(20-30$ p. 100$)$ ( $P O=1200$ mosm). Postfixation : $1 \mathrm{~h}$ au tétroxyde d'osmium (1-1,5 p. 100) dans la solution cacodylate. Inclusion : araldite ou épon 812.

b) Recherches cytochimiques. Recherche de polysaccharides : matériel inclus à I'araldite après double fixation ou simple fixation avec $\mathrm{OSO}_{4}$. Recherche de protéines : matériel inclus à l'épon après double fixation ou inclus au glycolmétacrylate (GMA) selon la technique de Leduc et Bernhard (1967), après fixation au glutaraldéhyde (5 p. 100).

2. Confection et coloration des coupes. - Coupes semi-fines et fines réalisées avec un ultramicrotome Jeol JUM 7 ou LKB UV. Coupes semi-fines colorées au bleu Unna ; coupes fines contrastées à l'acétate d'uranyle et au citrate de $\mathrm{Pb}$ (Reynolds, 1963) avant observation au microscope électronique Jeol $100 \mathrm{C}$.

3. Détection de protéines par action de protéases. - Les coupes d'animaux inclus au GMA ou à l'épon sont recueillies respectivement sur des grilles munies ou non d'une membrane de formvar. Les grilles sont mises à flotter par la face de support des coupes sur les solutions d'enzymes ou les solutions tampons correspondantes pour les témoins, à $37^{\circ} \mathrm{C}$, en atmosphère saturée de vapeur d'eau, pendant des temps compris entre 6 et $48 \mathrm{~h}$. Après chaque opération, les coupes sont rincées à plusieurs reprises sur les solutions tampons, puis sur de l'eau distillée, avant d'être contrastées. Solutions d'enzymes utilisées : pronase (Calbiochem, lyoph., B grade) en solution 0,1 p. $100,0,25$ p. 100 et 0,5 p. 100 dans de l'eau à $\mathrm{pH} 7,6$; trypsine (Calbiochem, cryst., A grade), en solution 0,5 p. 100 dans un tampon aux phosphates de Sörensen, $\mathrm{pH} 7,6$; pepsine (Calbiochem, $3 \alpha$ cryst., B grade) en solution 0,5 p. 100 dans $\mathrm{HCl} \mathrm{0,1} \mathrm{N}$. 
4. Détection de polysaccharides. - Les coupes fines, recueillies sur des grilles en or ou en nickel, sont traitées selon la technique à l'acide périodique thiocarbohydrazide - protéinate d'argent (PATAg) (Thiéry et Rambourg, 1974). La durée du traitement par la thiocarbohydrazide, après oxydation par l'acide périodique $0,1 \mathrm{p} .100$, varie entre $40 \mathrm{~min}$. et $65 \mathrm{~h}$. Les témoins correspondent à des coupes subissant le même traitement sauf l'oxydation préalable par l'acide périodique, remplacé par une solution $5 \mathrm{p} .100 \mathrm{~d}^{\prime} \mathrm{H}_{2} \mathrm{O}_{2}$ à 110 volumes.

\section{Résultats.}

Les étapes de l'ovogenèse chez Centropages typicus présentent un certain nombre d'analogies avec celles des Malacostracés; nous les décrirons en utilisant la terminologie proposée par Charniaux-Cotton (1978) et en insistant essentiellement sur leurs caractères les plus originaux.

\section{Topographie de l'ovaire.}

L'organisation de l'appareil génital femelle répond à celle décrite par Lowe (1935) pour Calanus finmarchicus. L'ovaire est médian et dorsal : sa partie postérieure, étroite, en contact avec le mésentère intestinal au niveau de la zone intermédiaire de l'intestin moyen (Arnaud et al. 1978), constitue la zone germinative où s'observent des ovogonies (fig. 1-3) ; sa partie antérieure est occupée par des ovocytes en préméiose et prévitellogenèse (fig. 2-4). Les phases ultimes de la croissance des ovocytes, ainsi que leur maturation, s'effectuent dans deux lobes latéro-dorsaux et latéro-ventraux postérieurs, largement soudés à l'ovaire (fig. 5), et interprétés comme les diverticules antérieurs et postérieurs des oviductes (Hilton, 1932 ; Razouls, 1981) ; les ovocytes les plus âgés y occupent toujours une position ventrale par rapport aux moins âgés (fig. 5-7). Des ovocytes matures ont été observés dans les diverticules postérieurs : leur taille est de $80 \mu \mathrm{m}$ environ et ils semblent bloqués en métaphase I (fig. 8), aucun globule polaire n'ayant été repéré. Chaque diverticule postérieur se termine au niveau du dernier segment thoracique et se continue par l'oviducte (fig. 8) jusqu'à l'orifice génital, situé sur le premier segment abdominal.

\section{Les phases de l'ovogenèse}

1. Phases germinative et de préméiose. - Les ovogonies (diamètre : 4 à $5 \mu \mathrm{m})$ montrent les caractères habituels de ce type cellulaire : noyau sombre, absence ou rareté des organites cytoplasmiques sauf des mitochondries, rapport nucléo-plasmique élevé. L'entrée en prophase méiotique (préméiose) (fig. 9) s'accompagne d'un accroissement sensible des cellules (diamètre : 5-8 $\mu \mathrm{m}$ ) et de l'apparition d'extrusions nucléaires.

2. Vitellogenèse primaire. - A partir du stade diplotène, l'enveloppe nucléaire acquiert un contour irrégulier; les pores deviennent de plus en plus nombreux et de grandes quantités d'extrusions nucléaires y transiteraient pour s'accumuler dans les concavités formées par l'enveloppe nucléaire. Le nucléole, unique, est volumineux (4 à $6 \mu \mathrm{m}$ ). Ces structures, caractéristiques de la prévitel- 

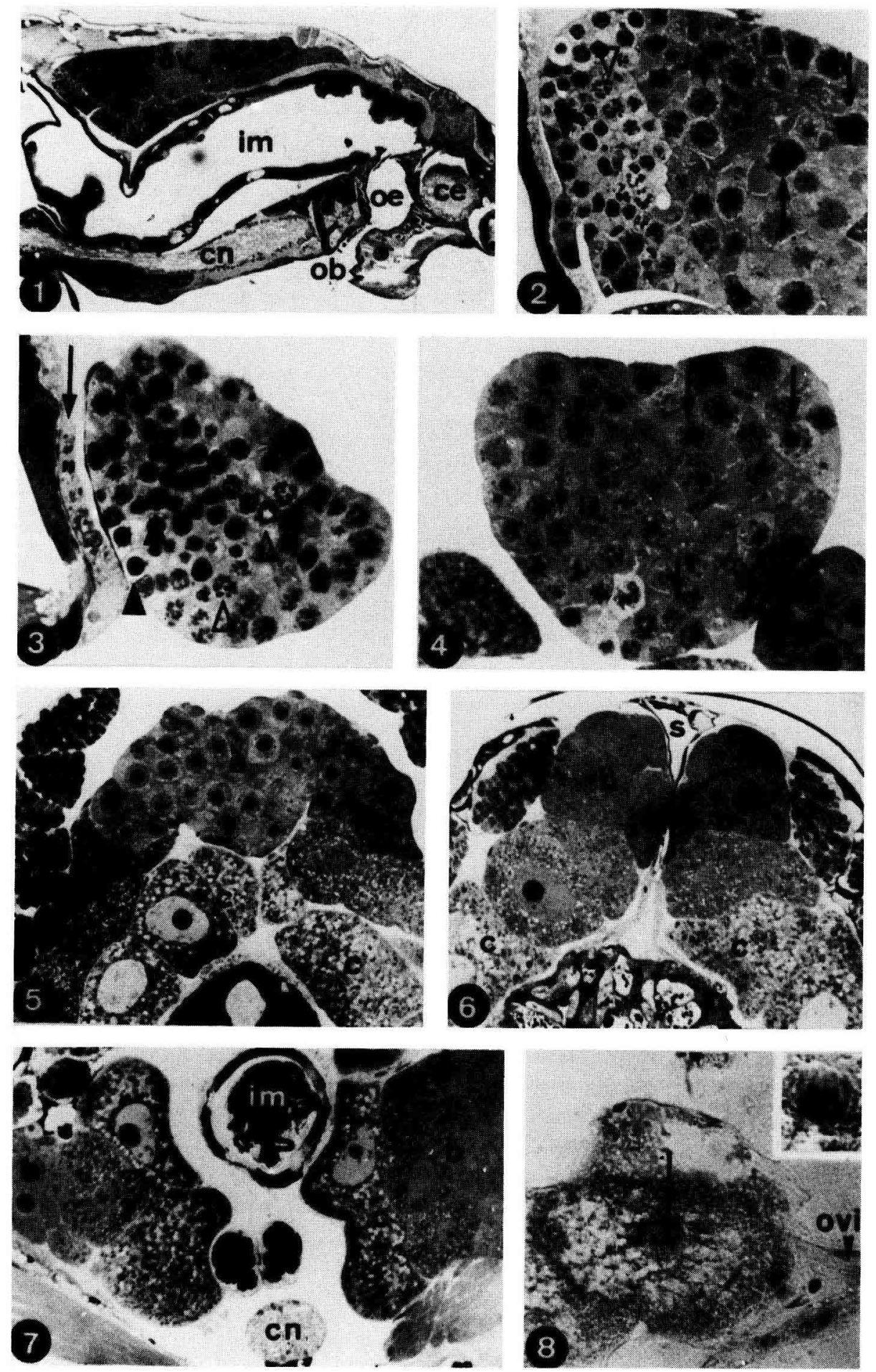
logenèse (fig. 10), ne changent guère au cours de toute la vitellogenèse primaire. Pendant cette phase, les mitochondries, dont le nombre s'accroît fortement, subissent diverses transformations intéressant leur taille, leur morphologie et leur distribution (fig. 10-12, 14). Le réticulum endoplasmique granulaire (REG) apparaît sous la forme de lamelles aplaties ou de petites vésicules renfermant quelques granules protéiques de $600 \AA$ environ (fig. 13) ; il se développe progressivement en un réseau de cavités assez polymorphes, remplies par ces petits granules; à leur voisinage s'observent parfois des dictyosomes de taille réduite (fig. 13). Les granules intracisternaux, qui représentent le vitellus endogène, restent distincts ou fusionnent en un matériel dense aux électrons ; les vésicules de REG, de 0,5 à $1 \mu \mathrm{m}$, qui le contiennent, correspondent donc à des grains de vitellus (fig. 15, 17-18). Vers la fin de la vitellogenèse primaire apparaissent des vacuoles claires, à contenu hétérogène, mesurant jusqu'à $1 \mu \mathrm{m}$ (fig. 16) ; le contenu des grains de vitellus, qui ont considérablement augmenté en nombre, présente souvent un aspect laqué (fig. 18). Au terme de cette phase, les ovocytes atteignent $30-40 \mu \mathrm{m}$, leur noyau $15-20 \mu \mathrm{m}$ et leur nucléole 6-10 $\mu \mathrm{m}$.

FIG. 1-33. - Abréviations utilisées: épithélium ovarien (EO), ovocyte en vitellogenèse primaire (OV1), ovocyte en vitellogenèse secondaire (OV2), chaîne nerveuse (cn), cerveau (ce), dictyosome (d), enclave lipidique (el), enveloppe nucléaire (en), extrusions nucléaires (exn), enveloppe vitelline (ev), granules intracisternaux (gi), grain de vitellus endogène (gv), globule vitellin exogène (glv), intestin moyen (im), invagination de l'oolemme (in), enclave lamellaire (la), mitochondrie $(\mathrm{m})$, matériel de l'hémolymphe $(\mathrm{mh})$, noyau $(\mathrm{n})$, nucléole (nu), orifice buccal (ob), oesophage (oe), ovaire (ov), oviducte (ovi), réticulum endoplasmique granulaire (reg), vacuole claire (vc), vacuole hétérogène (vh), vésicules de transition (vt).

FIG. 1-2. - Coupes sagittales semi-fines de Centropages typicus la portion proximale médio-dorsale de l'ovaire. $\times 95$. Fig. 2. Détail de cette portion de l'ovaire : dans la zone germinative, noter les ovogonies au repos (triangle noir) avec petit nucléole ou en division (triangle clair) avec chromosomes bien individualisés; les ovocytes en préméiose (flèche courte) et en prévitellogenèse (flèches longues) possèdent un nucléole toujours bien visible. $\times 490$.

FIG. 3. - Coupe transversale semi-fine de la zone germinative de l'ovaire. Noter les ovogonies au repos (triangles noirs) et en division (triangles clairs), ainsi que l'accolement de l'ovaire au mésentère intestinal (flèche). $\times 660$.

FIG. 4-6. - Coupes transversales semi-fines en avant de la zone germinative. Fig. 4. Zone ovarienne impaire médio-dorsale avec ovocytes en préméiose (flèches courtes) et en prévitellogenèse (flèches longues) $\times$ 490. Fig. 5. Zone de liaison entre l'ovaire impair médio-dorsal et les lobes latéro-dorsaux la : ovocytes en prévitellogenèse; b : ovocytes en vitellogenèse primaire ; $c$ : ovocytes en vitellogenèse secondaire). $\times 270$. Fig. 6. Lobes ovariens antérieurs séparés par les parois accolées (flèche) du sinus dorsal (s) (b et $\mathrm{c}:$ cf. fig. 5) $\times 270$.

FIG. 7. - Coupe transversale semi-fine en arrière de la zone germinative. (b et c : cf. fig. 5 ). $\times 240$.

FIG. 8. - Coupe histologique au niveau de l'oviducte. Noter la présence d'un ovocyte, bloqué en métaphase I (flèche) (fixation au liquide de Carnoy; inclusion à la paraffine ; coloration au vert de méthyle-pyronine). $\times 480$. Encart : fuseau de division et chromosomes métaphasiques d'un autre ovocyte. $\times 740$. 

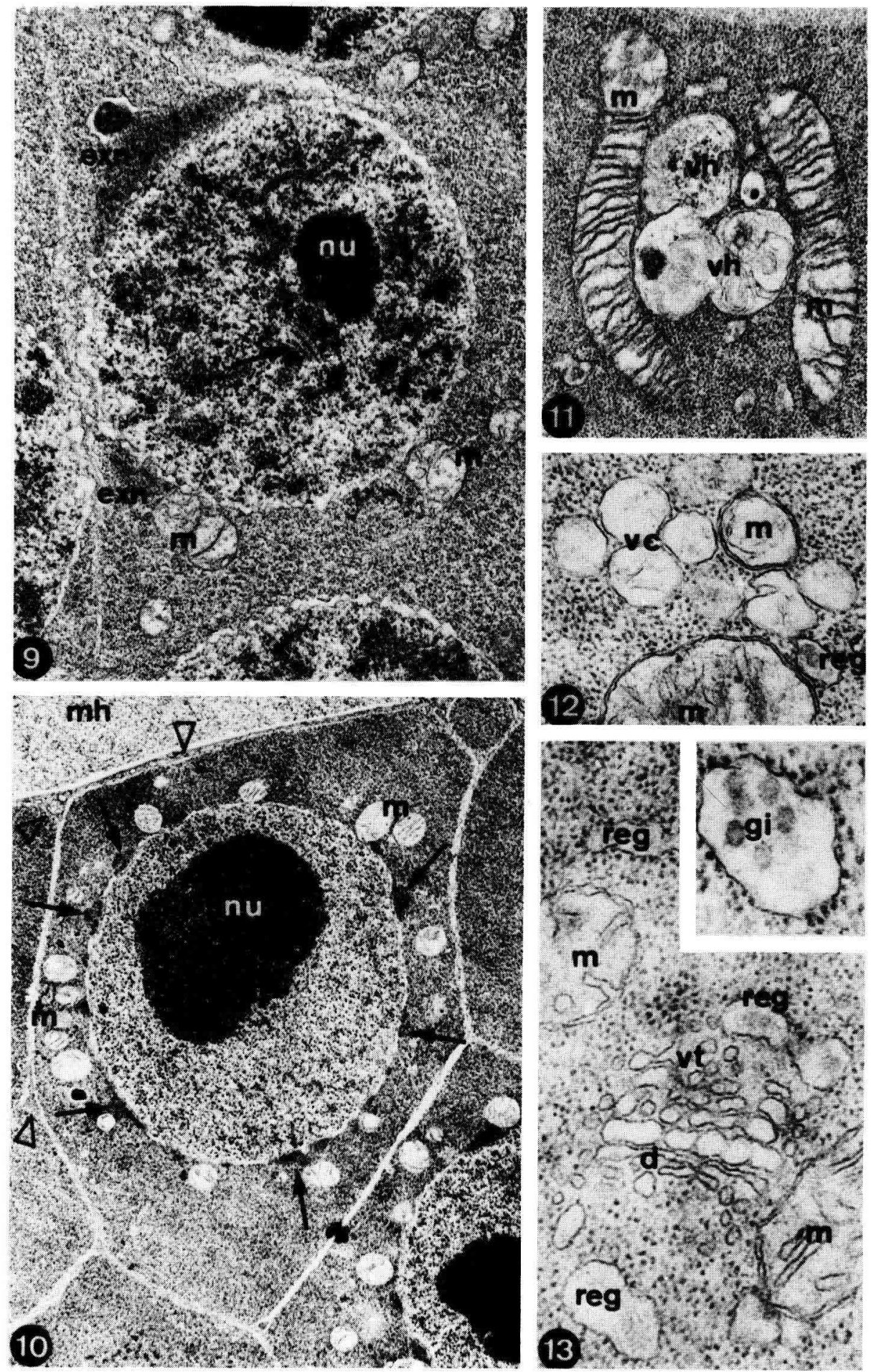
3. Vitellogenèse secondaire. - Le début de la vitellogenèse secondaire se marque par une nette augmentation de la taille des ovocytes due essentiellement à deux types d'événements :

a) L'apparition de petites inclusions de 500 à $1000 \AA$, denses et dépourvues de membrane (fig. 20), qui ne tardent pas à s'associer en masses plus volumineuses $(1,3-1,5 \mu \mathrm{m}$ de diamètre), offrant les caractères morphologiques de gouttelettes lipidiques (fig. 19, 21).

b) La formation d'une enveloppe vitelline de $0,1 \mu \mathrm{m}$ autour de l'ovocyte, coïncidant avec le développement brusque d'un autre type d'enclaves constituées par des globules vitellins de 1 à $4 \mu \mathrm{m}$ (fig. 19). L'enveloppe vitelline et I'oolemme dessinent souvent des replis dans l'ooplasme périphérique. Celui-ci renferme par ailleurs une multitude de petites vésicules de $0,1 \mu \mathrm{m}$ environ et l'oolemme montre fréquemment des invaginations de même diamètre (fig. 20). Enveloppe vitelline, vésicules et vacuoles ont une texture similaire, finement granulaire.

Ajoutons qu'en vitellogenèse secondaire, les vésicules de REG persistent, du vitellus endogène continuant ainsi à s'accumuler (fig. 21); peu à peu cependant le réticulum tend à devenir lisse, surtout au niveau des grains denses dont l'aspect laqué se généralise. Les vacuoles claires apparues en fin de vitellogenèse primaire disparaissent assez rapidement. Les dictyosomes conservent sensiblement le même aspect qu'en fin de vitellogenèse primaire. Le noyau forme une vésicule germinative de $20-25 \mu \mathrm{m}$; le nucléole n'est pas toujours bien visible, parfois complètement résorbé.

Vers la fin de la vitellogenèse secondaire, l'enveloppe vitelline se modifie ; son matériel présente une striation caractéristique (fig. 22, 23, 26) sur une épaisseur uniforme de $0,1 \mu \mathrm{m}$, sauf au niveau de certains points de contact entre l'oolemme et la membrane des cellules de l'épithélium ovarien (fig. 23). Quelques ovocytes montrent une nouvelle catégories d'enclaves sous forme de vésicules ovoïdes de 0,3-0,5 $\mu \mathrm{m}$ dont les plus évoluées renferment un matériel ordonné en lamelles plus ou moins parallèles (fig. 24-25). La taille finale des ovocytes est de l'ordre de $80 \mu \mathrm{m}$.

FIG. 9. - Ovocyte en préméiose à chromosomes visibles. Noter les mitochondries $(\mathrm{m})$ à matrice claire et crêtes rares et étroites, les extrusions nucléaires (exn), le complexe synaptonématique (flèche) et le nucléole (nu). $\times 10000$.

FIG. 10. - Ovocyte en prévitellogenèse. L'aspect clair du nucléoplasme indiquant la fin de la préméiose, le nucléole (nu) volumineux, les organites représentés seulement par des mitochondries $(\mathrm{m})$ au contact d'abondantes extrusions nucléaires (flèches) caractérisent cette phase. Séparant plusieurs ovocytes, on observe aussi de fins prolongements de l'épithélium ovarien Itriangles. $\times 4200$.

FIG. 11-13. - Portions d'ovocytes en vitellogenèse primaire. Fig. 11. - Lors de la prévitellogenèse, les mitochondries $(\mathrm{m})$, souvent de grande taille (jusqu'à $4 \mu \mathrm{m}$ ), à crêtes étroites et parallèles, se regroupent fréquemment autour de vacuoles à contenu hétérogène (vh). $\times 16000$. Fig. 12. En début de vitellogenèse primaire, noter les amas de vacuoles claires (vc) pouvant résulter de la dégénérescence et du morcellement d'une ou plusieurs mitochondries. $\times 28000$. Fig. 13. Noter la présence de cavités de REG (reg) en cours de développement et d'un dictysome (d) produisant des vésicules claires. $\times 40000$. Encart. Vésicule de REG montrant plusieurs granules intracisternaux (gi). $\times 56000$. 

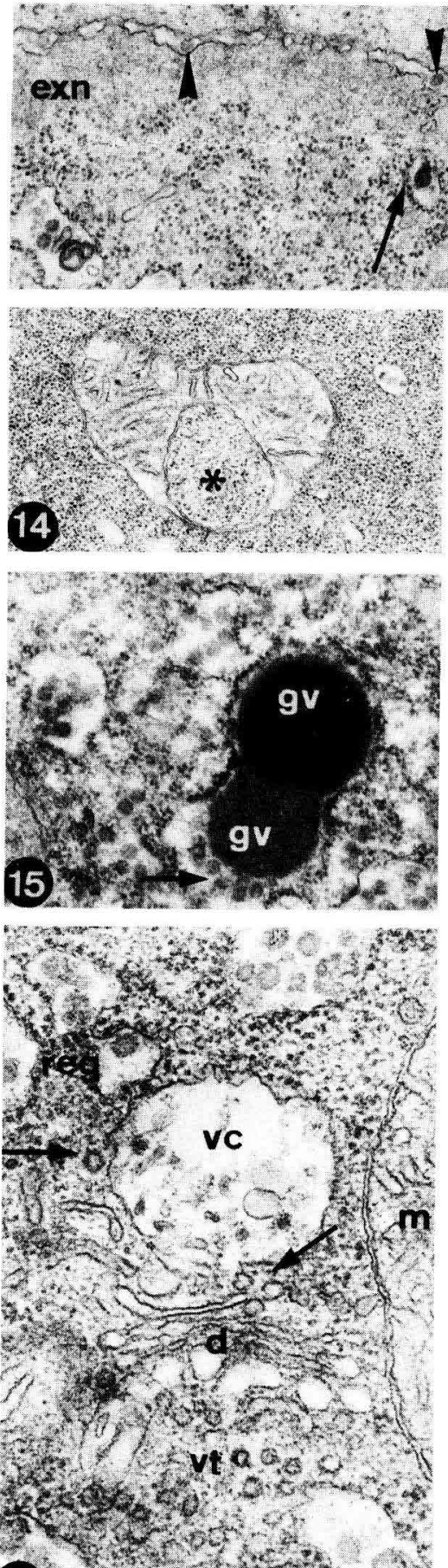

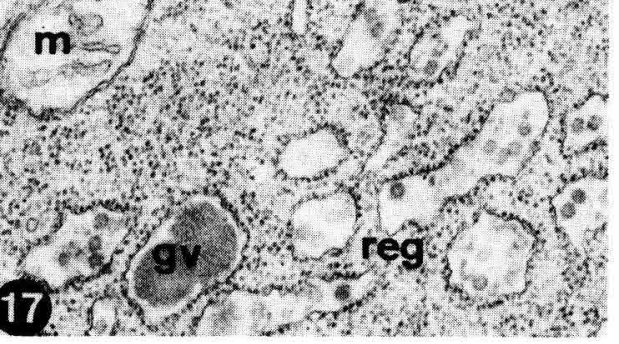

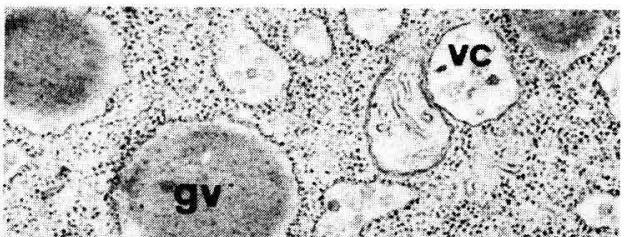

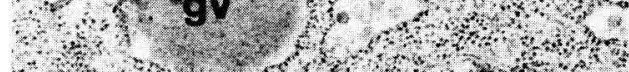
1.6.

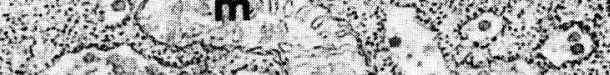

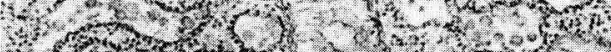

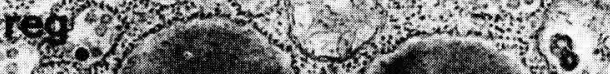

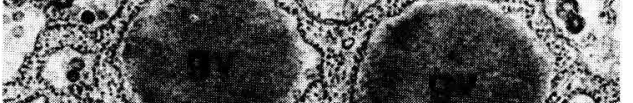

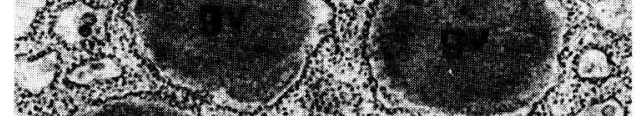
\%

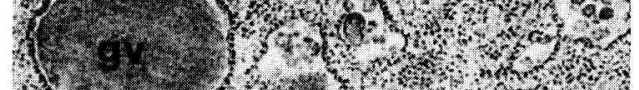
(2)

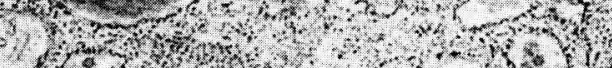

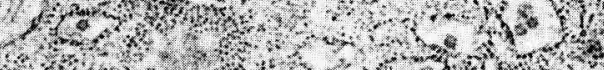

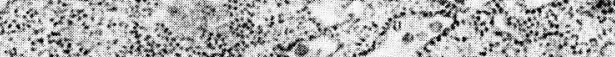

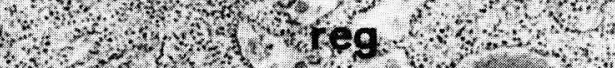

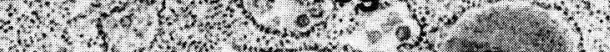

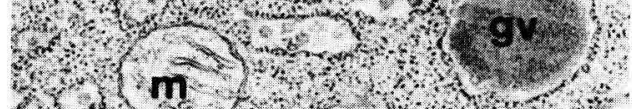

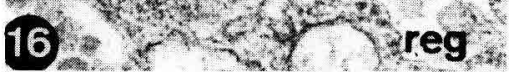
18 . 


\section{Modifications de l'épithélium ovarien au cours de l'ovogenèse.}

L'ovaire est entouré par un épithélium, doublé extérieurement d'une membrane basale à laquelle s'accole parfois le matériel dense, finement granulaire fréquemment contenu dans I'hémolymphe (fig. 19). Cet épithélium est mince et sombre au niveau des jeunes ovocytes - zones germinative, de préméiose et de prévitellogenèse essentiellement (fig. 10) -, plus large et clair au niveau des ovocytes plus âgés entre lesquels il envoie des prolongements étroits (fig. 19) ; ces ovocytes se trouvent donc complètement entourés par des cellules qu'on peut assimiler, à partir de ce stade, aux cellules folliculaires des Malacostracés. Les membranes contiguës des cellules épithéliales et des ovocytes sont séparées par un espace intercellulaire de largeur normale (150 à $250 \AA)$, qui persiste en certains points après l'apparition de l'enveloppe vitelline (fig. 23). Aucun dispositif particulier de jonction n'a été observé à leur niveau. C'est autour des ovocytes en vitellogenèse secondaire que les cellules de l'épithélium sont les plus développées ; on y relève souvent des enclaves denses (fig. 27). En fin de vitellogenèse secondaire, ces cellules semblent se dissocier pour libérer les ovocytes.

\section{Résultats cytochimiques.}

1. Recherche des protéines. - Après inclusion à l'épon, à partir d'un temps d'action de $13 \mathrm{~h}$ de la pronase, dans les ovocytes en vitellogenèse primaire, des grains de vitellus sont éclaircis, parfois évidés (fig. 28, 29); sur le même spécimen, de telles figures sont rares ou absentes après action de la pepsine (fig. 30) ou de la trypsine; sur d'autres animaux, enfin, aucun effet $n$ 'est visible, quelle que soit l'enzyme utilisée, même si l'action est prolongée jusqu'à 24 ou $48 \mathrm{~h}$. Après inclusion au GMA, l'effet des protéases se traduit par un éclaircissement généralisé, sauf au niveau des grains denses de vitellus endogène (fig. 31) dont le contraste tend à s'accentuer par rapport à des préparations non traitées (fig. 32) : dans les tests à la pepsine et à la trypsine, réalisés sur certains spécimens, il y a une perte quasi totale de contraste du hyaloplasme et des vésicules de vitellus exogène à partir d'un temps d'action de $24 \mathrm{~h}$ (fig. 31); les tests à la pronase, réalisés sur d'autres animaux, montrent un effet beaucoup plus atténué.

FIG. 14-15. - Portions d'ovocytes en vitellogenèse primaire. Fig. 14. Au cours de cette phase les amas mitochondriaux (cf. fig. 11-12) tendent à disparaître; on observe seulement des mitochondries isolées souvent incurvées et emprisonnant du matériel hyaloplasmique (astérique). $\times 22000$. Fig. 15. Observer la fusion de deux grains de vitellus d'aspect différent (gv), dont l'un contient encore des granules périphériques intacts (flèche). $\times 27000$.

FIG. 16-18. - Portions d'ovocytes en fin de vitellogenèse primaire. Fig. 16. Observer une vacuole claire (vc) avec des vésicules golgiennes (flèches) à son voisinage. $\times 42000$. Fig. 17. Dans la région périnucléaire, noter l'aspect des extrusions (exn), plus claires que le hyaloplasme, les lamelles ergastoplasmiques en relation probable avec l'enveloppe nucléaire (flèches), la présence de granules dans l'enveloppe nucléaire (triangles). $\times 29000$. Fig. 18. Au terme de la vitellogenèse primaire, les vésicules de REG contenant des granules ou des grains de vitellus (gv) sont nombreuses ; le matériel de ces grains est plus contrasté que celui des granules et présente un aspect laqué. Noter la persistance des ribosomes sur l'enveloppe des grains. $\times 22500$. 

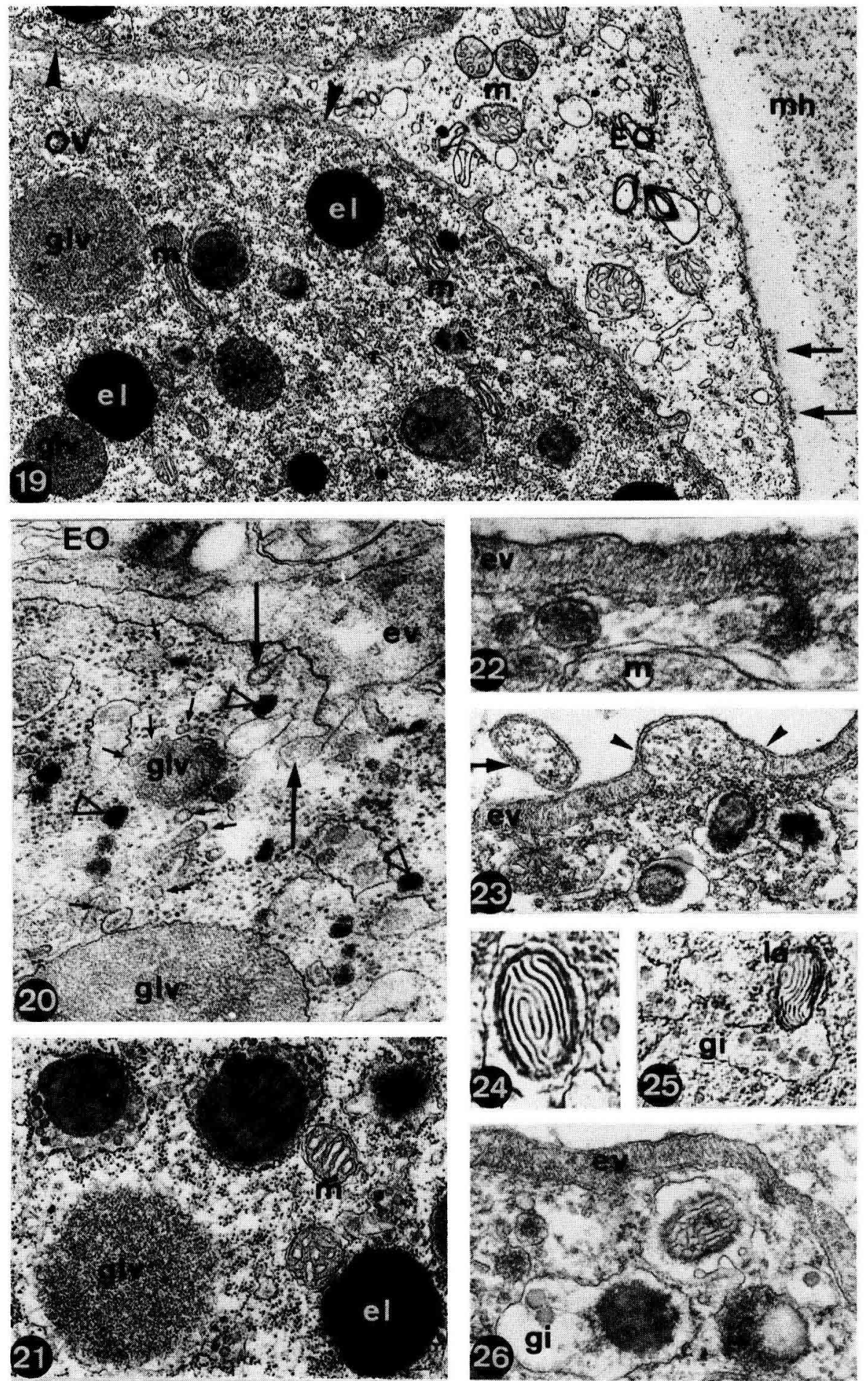


\section{Recherche des lipides et des polysaccharides.}

a) Les inclusions très osmiophiles, de plus en plus volumineuses, observées dans les ovocytes en cours de vitellogenèse secondaire, sont interprétées comme étant de nature lipidique. Cette hypothèse a été confirmée par la réaction au noir Soudan $\mathrm{B}$, réalisée sur des coupes à la paraffine et fortement positive dans tous les ovocytes âgés.

b) La technique du PATAg ne fournit d'images contrastées qu'au niveau des ovocytes en vitellogenèse secondaire ; à ce stade, des dépôts d'Ag sont toujours présents sous forme de grains plus ou moins dispersés dans le hyaloplasme (fig. 33) ou groupés en amas volumineux.

\section{Discussion.}

Ces données sur le déroulement de l'ovogenèse chez les Copépodes mettent en évidence la double origine du vitellus, endogène et exogène, bien connue chez les Malacostracés.

\section{Vitellogenèse primaire.}

La vitellogenèse primaire, au cours de laquelle s'édifie le vitellus endogène, débute par une phase de prévitellogenèse dont les manifestations sont maintenant

FIG. 19-20. - Portions d'ovocytes en vitellogenèse secondaire. Fig. 19. Remarquer les différents types d'enclaves: grains de vitellus endogène (gv), d'aspect laqué, enclaves lipidiques (el), homogènes et très denses, globules vitellins (glv), à texture finement granulaire. Noter aussi les mitochondries $(\mathrm{m})$ à matrice dense, la présence de l'enveloppe vitelline (triangles) autour des ovocytes (OV2), le développement de l'épithélium ovarien (EO) et le matériel finement granulaire contenu dans I'hémolymphe (mh) et parfois accolé à la lame basale (flèches) des cellules épithéliales. $\times 5000$. Fig. 20. Dans l'ooplasme cortical, relever les invaginations (flèches longues) de l'oolemme, les petites vésicules (flèches courtes) au voisinage des globules vitellins (glv) et les inclusions lipidiques (triangles). $\times 45000$.

FIG. 21. - On observe avec plus de détails que sur la fig. 19 les 3 types d'enclaves ooplasmiques. Noter aussi l'enveloppe granulaire et la présence de granules autour du vitellus endogène d'aspect laqué, ainsi que la discontinuité de la membrane du globule vitellin (glv), phénomène assez fréquemment observé. $\times 20000$.

FIG. 22-26. - Ooplasme cortical d'ovocytes en fin de vitellogenèse secondaire. Fig. 22. Relever la structure périodique de l'enveloppe vitelline (ev) et la persistance de petites invaginations de I'oolemme à contenu dense. $\times 66000$. Fig. 23. Noter la présence de vésicules contenant un matériel dense et l'interruption locale de l'enveloppe vitelline (triangles). L'épithélium ovarien à ce stade est constitué par des cellules très évidées, renfermant des vacuoles à double membrane (flèche), qui pourraient provenir d'un rejet de matériel ovocytaire au niveau des zones de contact avec l'oolemme.

FIG. 24-25. - Les dernières enclaves ooplasmiques à apparaître ont un contenu décollé de la membrane et présentent une enveloppe fibreuse entourant un matériel dense, ordonné en lamelles plus ou moins parallèles. $\times 45000$ (fig. 24) et $\times 28000$ (fig. 25). Fig. 26. Les enclaves lamellaires semblent résulter de la réorganisation secondaire d'un matériel dense, contenu dans des vésicules corticales observées précédemment (cf. fig. 23). $\times 32000$. 

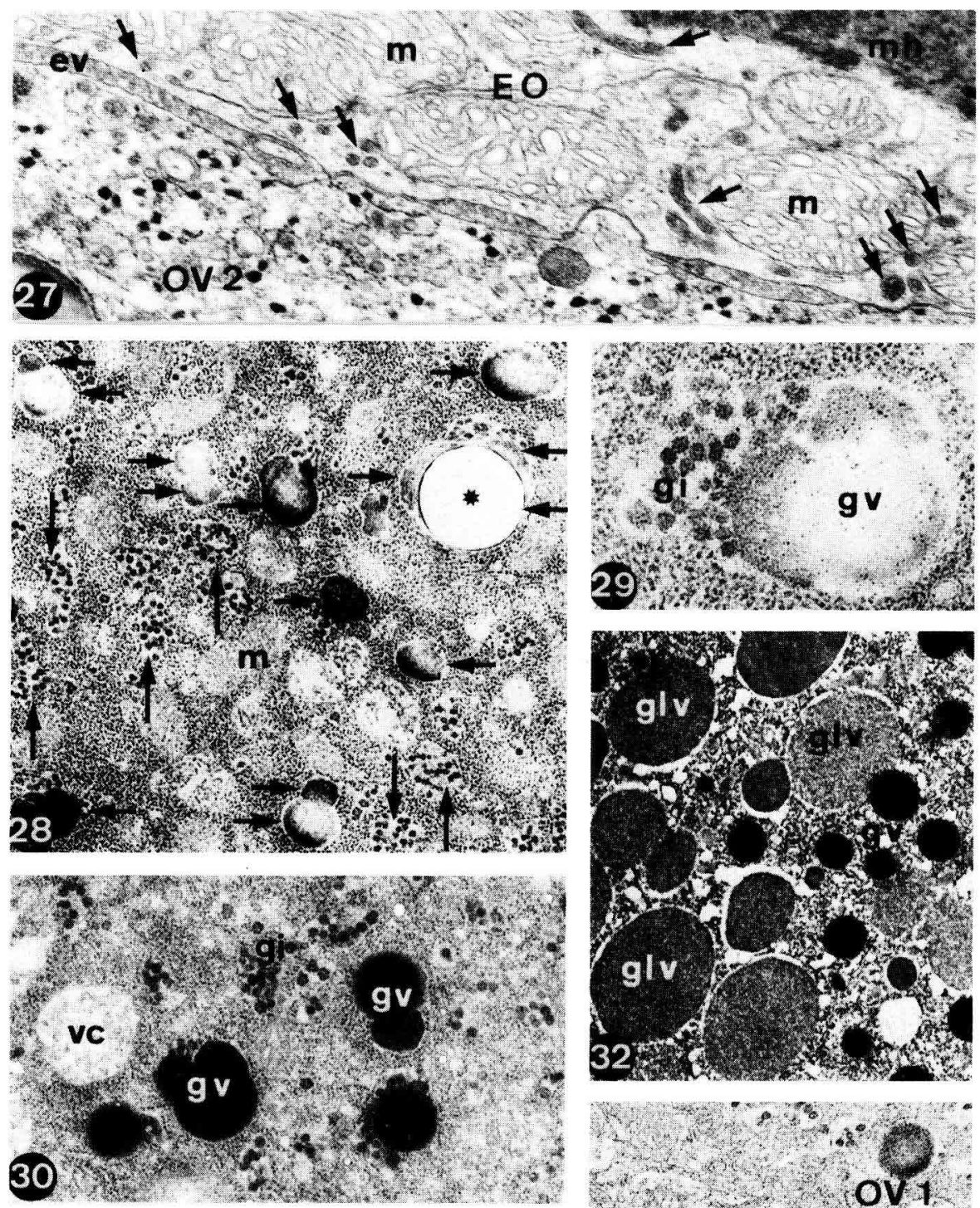

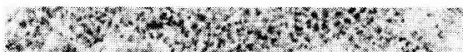

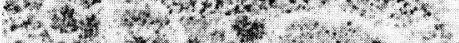

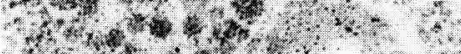

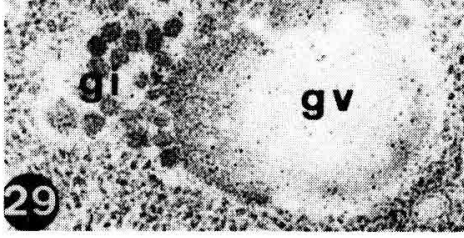
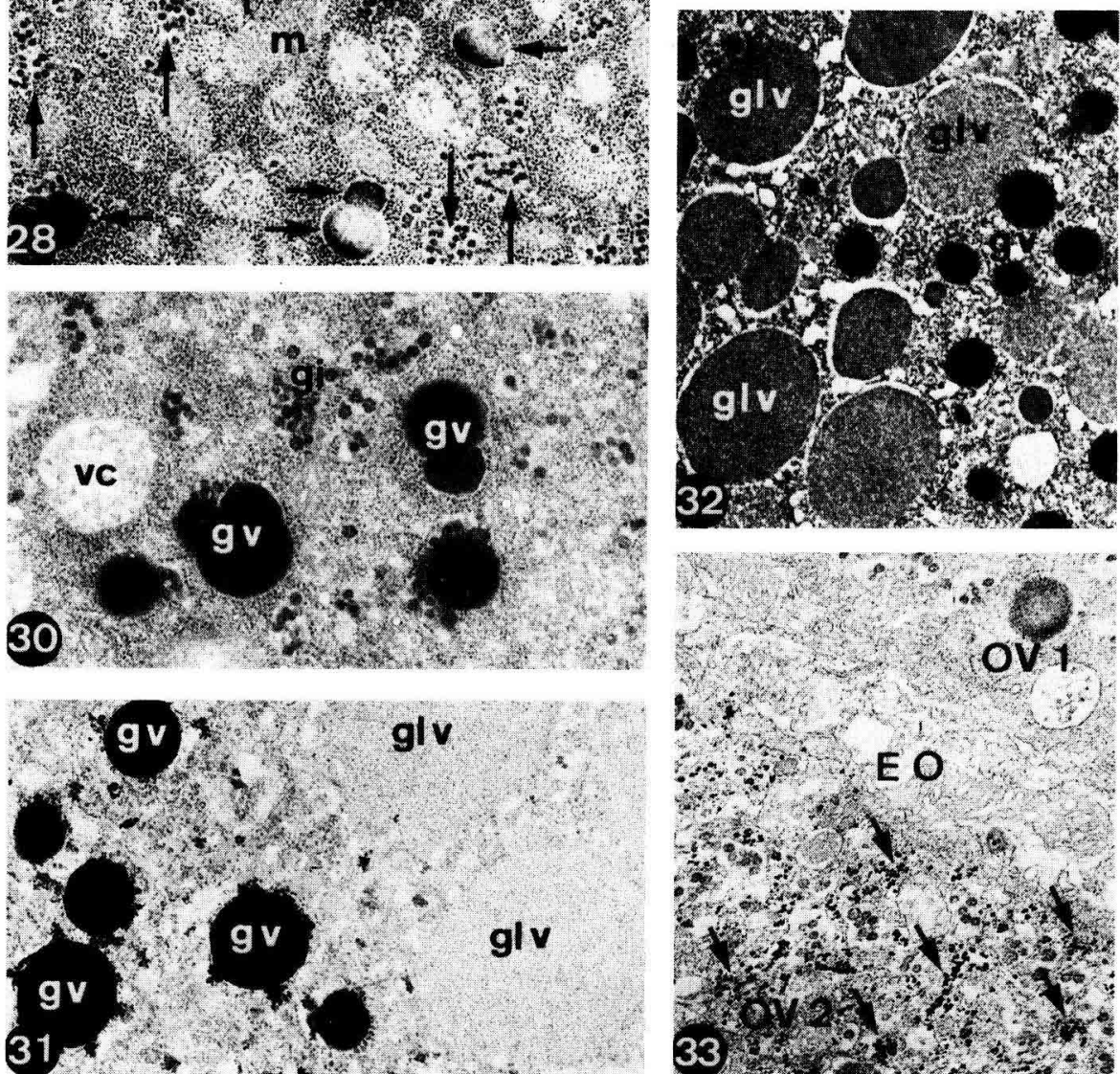
classiques. Le comportement original des mitochondries, observé au cours de cette phase - disposition périnucléaire et amas mitochondriaux notamment - est vraisemblablement en relation avec l'accroissement et la multiplication de ces organites, phénomène suggéré chez d'autres espèces animales par plusieurs auteurs, Clérot (1976) et Callen et al. (1980) en particulier.

La vitellogenèse proprement dite débute avec le développement du REG et l'accumulation dans ses cavités de granules denses qui fusionnent ou non entre eux et représentent le vitellus endogène. Des accumulations assez semblables, au niveau du REG, sont relatées chez un Cladocère du genre Daphnia (Kessel, 1968) et chez la quasi-totalité des Malacostracés étudiés, Amphipodes (Orchestia gammarellus) par Zerbib (1973, 1978, 1980), Isopodes (/dotea balthica basteri) par Souty (1980) et surtout Décapodes : Astacus (Beams et Kessel, 1962, 1963 ; Ganion et Kessel, 1972 ; Zerbib, 1979), Homarus (Kessel, 1968), Libinia (Hinsch et Cone, 1969), Cancer (Eurenius, 1973) et Eriocheir (Dhainaut et de Leersnyder, 1976). Dans plusieurs cas, on a démontré que le matériel des vésicules ergastoplasmiques était de nature protéique ou glycoprotéique (Dhainaut et de Leersnyder, 1976 ; Zerbib, 1976 ; Kessel, 1968) ou même lipoglycoprotéique (Erribabu et al., 1978 ; Varadarajan et Subramoniam, 1980). Chez Centropages typicus, les tests de réaction enzymatique n'ont pas montré de relations significatives entre la durée du test, le caractère plus ou moins hydrosoluble du milieu d'inclusion et

FIG. 27. - Portion d'épithélium ovarien bordant un ovocyte en vitellogenèse secondaire. Noter les enclaves intraépithéliales (flèches), de même texture que le matériel présent dans I'hémolymphe $(\mathrm{mh})$ et au niveau de l'enveloppe vitelline (ev). $\times 22000$.

FIG. 28-29. - Action de la pronace (0,5 p. 100), 13 h) sur des ovocytes en vitellogenèse primaire après inclusion à l'épon. Fig. 28. Les grains de vitellus (flèches courtes) sont attaqués à des degrés divers, entraînant parfois l'éclatement de la résine (astérisque) ; les granules intracisternaux (flèches longues), intacts, tendent au contraire à présenter un contraste plus accentué. $\times 10000$. Fig. 29. Noter l'éclaircissement pratiquement limité à l'un des 3 grains en cours de coalescence. $\times 33000$.

FIG. 30. - Action de la pepsine (0,5 p. 100, $13 \mathrm{~h})$ sur des ovocytes en vitellogenèse primaire après inc/usion à l'épon. $\times 14000$.

FIG. 31. - Action de la pepsine 10,5 p. 100, $24 \mathrm{~h})$ sur des ovocytes en vitellogenèse secondaire après inclusion au GMA. × 65000 .

FIG. 32. - Ovocyte en vitellogenese secondaire après inclusion au GMA. $\times 42000$.

FIG. 33. - Test PATAg sur des ovocytes en vitellogenèse primaire et en vitellogenèse secondaire après inc/usion à l'ara/dite. Test PATAg: acide périodique, $25 \mathrm{~mm}$; $\mathrm{TCH}, 60 \mathrm{~h}$; protéinate d'Ag, $30 \mathrm{mn}$. Le marquage (flèches) dans l'ovocyte en vitellogenèse secondaire est dû à la réaction entre la $\mathrm{TCH}$ et les lipides insaturés osmiés après oxydation par l'acide périodique; aucun dépôt ne figure jamais dans les ovocytes en vitellogenèse primaire, ni dans les ovocytes âgés de préparations témoins traitées par $\mathrm{H}_{2} \mathrm{O}_{2}$ dont l'action est insuffisante sur les lipides osmiés pour permettre une réaction avec la $\mathrm{TCH}$. 
l'intensité de l'attaque enzymatique. Seuls les grains de vitellus de certains ovocytes en vitellogenèse primaire, après inclusion à l'épon, subissent une digestion notable sous l'action de la pronase; par contre, dans les ovocytes testés après inclusion au GMA, ainsi que dans les ovocytes en vitellogenèse secondaire après inclusion à l'épon, le vitellus endogène n'est pas attaqué. II peut s'agir là d'un résultat faussement négatif, explicable par une association plus ou moins précoce de protéines avec d'autres substances ; cette hypothèse semble concrétisée, au niveau ultrastructural, par la fréquence des grains de vitellus montrant une nette dualité de leur matériel (fig. 15) ; d'ailleurs, en vitellogenèse secondaire, les grains sont toujours homogènes et possèdent un aspect " laqué " caractéristique laissant présumer une association avec des lipides (fig. 21). Les grains matures de vitellus endogène pourraient donc représenter un complexe lipoprotéique ou lipoglycoprotéique très dense, résistant notamment aux enzymes protéolytiques, à la façon des constituants membranaires. Les différences notées selon les individus concernant l'action des protéases peuvent traduire aussi des variations intervenant dans la composition et la structure chimiques du vitellus de ces grains.

Les vacuoles claires observées en fin de vitellogenèse primaire n'ont pas une origine nettement élucidée. La majorité d'entre elles, sinon la totalité, représentent sans doute des autophagosomes dont le contenu hétérogène provient soit de la lyse de matériel ooplasmique séquestré, soit de mitochondries en dégénérescence, soit encore de l'involution de vésicules ergastoplasmiques à granules. A l'appui de cette hypothèse, on note que certaines structures apparaissent comme des stades intermédiaires entre vacuoles et mitochondries ou vésicules ergastoplasmiques typiques ; par ailleurs, de telles formations augmentent considérablement en nombre chez des animaux soumis à un jeûne prolongé. Ces vacuoles disparaissent toutes au cours de la vitellogenèse secondaire.

L'appareil de Golgi montre une activité relativement importante, mais, en raison de l'absence de tout grain de sécrétion, son rôle reste hypothétique. II remplit vraisemblablement plusieurs fonctions pendant la vitellogenèse. L'aspect de certaines structures golgiennes et péri-golgiennes, dès le début de la vitellogenèse primaire, laisse présager que les nombreuses petites vésicules claires formées fusionnent avec le REG préexistant. L'originalité reconnue de l'appareil de Golgi réside dans ses propriétés de glycosylation et sulfatation d'accepteurs variés. Dans le cas présent, il pourrait assumer ce rôle pour achever la synthèse soit des protéines et des lipides membranaires du réticulum, organite dont le développement est particulièrement important au cours de cette période, soit du matériel vitellin élaboré par le REG selon un cycle d'activité en circuit fermé "Réticulum $\rightarrow$ Golgi $\rightarrow$ Réticulum », hypothèse plausible, puisque chez les Copépodes et les Crustacés en général (Kessel, 1968 ; Anderson, 1974), le réticulum assure le stockage du vitellus endogène. Par ailleurs, l'appareil de Golgi pourrait emmagasiner des hydrolases intervenant dans les processus d'autophagie évoqués précédemment, comme semblent l'indiquer certaines figures (fig. 16). De manière générale, le rôle de l'appareil de Golgi dans la vitellogenèse des Crustacés est sujet à controverse : pour certains (Hinsch et Cone, 1969 ; Eurenius, 1973 ; Dhainaut et de Leersnyder, 1976 ; Beams et Kessel, 1980), les 
sécrétions golgiennes interviendraient à des degrés divers dans la formation du vitellus, tandis que pour d'autres, elles n'auraient aucun rôle direct (Bilinski, 1979).

\section{Vitellogenèse secondaire.}

Elle correspond, pour l'essentiel, à d'importantes accumulations de substances exogènes sous la forme de globules vitellins granulaires et d'enclaves denses de forme variable.

a) Les enclaves réparties dans le hyaloplasme représentent des gouttelettes lipidiques, comme le prouvent leur aspect en microscopie électronique et la réaction positive au noir Soudan B observée dans les ovocytes âgés. Leur opacité, due à l'acide osmique, indique la présence de composants insaturés responsables des dépôts d'argent importants observés dans les ovocytes en vitellogenèse secondaire avec les tests au PATAg (fig. 33). II est à noter que l'aspect des grains d'argent est différent de celui de particules de glycogène dont l'absence est confirmée par toutes les observations réalisées sur des ovocytes après simple fixation à l'acide osmique. L'apparition des lipides, sous la forme de petites inclusions transitant à travers l'oolemme (fig. 20), constitue la première manifestation de la vitellogenèse secondaire. Pour une part importante, ces lipides peuvent provenir des réserves accumulées dans le mésentère et dont la diminution est notable au cours de l'ovogenèse.

b) Quant aux globules vitellins, ils apparaissent dès la mise en place de l'enveloppe vitelline. De nombreuses vésicules sont alors présentes au voisinage de l'oolemme et montrent de fréquentes figures de fusion avec des globules en formation dont le contenu est de même texture que l'enveloppe vitelline. Ces observations tendent à prouver l'origine exogène de ce vitellus aux dépens du matériel de l'enveloppe dont la pénétration se ferait par endocytose, comme chez les Malacostracés. Les clichés obtenus (fig. 20) ressemblent en effet à tous ceux publiés par de nombreux auteurs chez les Crustacés : Hinsch et Cone (1969) pour Libinia, Wolin et al. (1973) pour Uca, Libinia et Cambarus, Dhainaut et de Leersnyder (1976) pour Eriocheir, Zerbib pour Orchestia gammarellus (1973, 1978), Astacus astacus et A. leptodactylus (1979), Bilinski (1979) pour Porcellio scaber, Beams et Kessel (1980) pour Oniscus ase/lus. Le net éclaircissement des globules vitellins chez Centropages typicus après action des protéases (fig. 31) prouve l'existence de protéines ; par ailleurs, leur aspect et leur faible contraste, différents de ceux observés chez les Malacostracés, ne semblent pas indiquer la présence de lipides associés. Leur composition pourrait être de nature glycoprotéique. Chez les Malacostracés, le vitellus exogène est constitué par une lipoglycocaroténoprotéine, la vitellogénine ou VTG, qui est produite en dehors de l'ovaire, dans un lieu encore indéterminé pour la plupart des Crustacés (Meusy, 1980). Récemment toutefois, l'emploi de méthodes immunohistochimiques et immunoautoradiographiques a permis de montrer que chez Orchestia gammare/Ius (Croisille et Junéra, 1980), Porcellio dilatatus (Picaud et Souty, 1980 a et b) et Idotea balthica basteri (Souty et Picaud, 1981) le tissu adipeux sousépidermique correspondrait au site de synthèse de la vitellogénine. Chez les femelles de Centropages typicus en vitellogenèse secondaire, il se développe à la 
périphérie du corps, notamment autour de la chaîne nerveuse, un syncytium très dense, riche en REG et en formations golgiennes géantes, donnant naissance à de nombreux grains de sécrétion de densité moyenne. Ce tissu participe peutêtre à l'élaboration du vitellus exogène, qui serait ensuite apporté à l'ovaire par I'hémolymphe ; il est intéressant de noter à ce sujet que les femelles, à cette période, présentent souvent des espaces lacunaires ou sinusaires remplis d'un matériel dense, de texture assez semblable à celle de l'enveloppe vitelline (fig. 19, 27). Les enclaves de même densité observées dans (ou entre) les cellules de l'épithélium ovarien entourant les ovocytes âgés (fig. 27) pourraient représenter la forme de transit de ce matériel jusqu'à l'oolemme.

L'origine et la signification des formations à lamelles denses, signalées dans des ovocytes parvenus à maturité, est incertaine. Plusieurs clichés font penser qu'elles prennent naissance au niveau du réticulum (fig. 25, 26). La rareté des ovocytes montrant ces grains lamellaires n'a pas permis de pratiquer de tests histochimiques. Toutefois, leur structure, qui rappelle celle signalée par Zerbib (1975) pour les granules corticaux d'Orchestia gammarellus, et leur apparition tardive au cours de l'ovogenèse suggèrent qu'il pourrait s'agir aussi de futurs granules corticaux en cours de mise en place dans l'ooplasme périphérique. Ces granules n'auraient donc pas une origine golgienne directe. Cette interprétation rejoint les observations de plusieurs auteurs chez les Crustacés et d'autres Invertébrés indiquant l'intervention de matériel ayant pour origine le réticulum dans l'élaboration de la membrane de fécondation (Busson-Mabillot, 1969 ; Garreau de Loubresse, 1975 ; Goudeau et Lachaise, 1980).

\section{Conclusion.}

Des données développées précédemment, il ressort que, dans ses grandes lignes, l'ovogenèse de Centropages typicus ressemble par beaucoup d'aspects à celle des Malacostracés; la comparaison avec d'autres Entomostracés s'avère plus difficile, en raison de l'insuffisance des travaux portant sur ce groupe. La vitellogenèse primaire donne naissance à des grains de vitellus endogène très denses, constitués de protéines élaborées au niveau du REG, associées probablement à une fraction lipidique. La vitellogenèse secondaire, comme chez les Malacostracés, correspond aux accumulations les plus importantes de substances de réserve, d'origine exogène, sous la forme d'enclaves lipidiques et de globules vitellins ; le faible contraste et la sensibilité de ces derniers à l'action des protéases indiquent une nature glycoprotéique. Les enclaves lipidiques proviennent des volumineux dépôts de graisse stockés dans le mésentère intestinal et qui diminuent très fortement au cours de la vitellogenèse ; par contre, l'origine des substances des globules vitellins, qui pénètrent dans les ovocytes par endocytose, n'est pas élucidée. Comme chez les Malacostracés, où on les désigne sous le nom de vitellogénine, il est seulement certain que ces substances ont une origine extra-ovarienne et sont acheminées jusqu'à l'ovaire par l'hémolymphe. Chez Centropages typicus, un tissu syncytial périphérique, particulièrement développé au cours de la vitellogenèse, constitue un lieu de synthèse présumé. Enfin, cette 
étude confirme que le réticulum est le lieu d'élaboration et de stockage des grains de vitellus endogène ; en outre, il pourrait correspondre au site de différenciation d'enclaves à structure lamellaire caractéristique, apparaissant en fin de vitellogenèse secondaire et représentant peut-être les granules corticaux.

Reçu en octobre 1981.

Accepté en décembre 1981.

\section{Références}

ANDERSON E., 1974. Comparative aspects of the ultrastructure of the female gamete. Int. Rev. Cytol., Suppl. 4, 1-70.

ANTEUNIS A., FAUTREZ-FIRLEFYN N., FAUTREZ J., 1966 a. La structure des ponts intercellulaires "obturés " et " ouverts" entre oogonies et oocytes dans l'ovaire d'Artemia salina. Arch. Biol., $7,645-664$.

ANTEUNIS A., FAUTREZ-FIRLEFYN N., FAUTREZ J., 1966 b. A propos d'un complexe tubulomitochondrial ordonné dans le jeune ovocyte d'Artemia salina. J. ultrastr. Res., 15, 122-130.

ARNAUD J., BRUNET M., MAZZA J., 1978. Studies on the midgut of Centropages typicus (Copepod, Calanoid). I. Structural and ultrastructural data. Cell Tiss. Res., 187, 333-353.

ARNAUD J., BRUNET M., MAZZA J., 1980. Structure et ultrastructure comparées de l'intestin chez plusieurs espèces de Copépodes Calanoides (Crustacea). Zoomorphology, 95, 213-233.

BEAMS H. W., KESSEL R. G., 1962. Intracisternal granules of the endoplasmic reticulum in the crayfish oocyte. J. Cell Biol., 13, 158-162.

BEAMS H. W., KESSEL R. G., 1963. Electron microscope studies on developing crayfish oocytes with special references to the origin of yolk. J. Cell Biol., 18, 621-649.

BEAMS H. W., KESSEL R. G., 1980. Ultrastructure and vitellogenesis in the oocyte of the Crustacean, Oniscus asellus. J. submicrosc. Cytol., 12, 17-27.

BILINSKI S., 1979. Ultrastructural study of yolk formation in Porcellio scaber Latr. (Isopoda). Cytobios, 26, 123-130.

BUSSON-MABILLOT S., 1969. Données récentes sur la vitellogenèse. Ann. Biol., 8, 199-228.

CALLEN J. C., TOURTE M., DENNEBOUY N., MOUNOLOU J. C., 1980. Mitochondrial development in oocytes of Xenopus laevis. Biol. cell., 38, 9-12.

CHARNIAUX-COTTON H., 1978. L'ovogenèse, la vitellogenèse et leur contrôle chez le Crustacé Amphipode Orchestia gammarellus (Pallas). Comparaison avec d'autres Malacostracés. Arch. Zool. Exp. gen., 119, 365-397.

CLÉROT J. C., 1976. Les groupements mitochondriaux des cellules germinales des Poissons Téléostéens Cyprinidés. I. Etude structurale. J. uttrastr. Res., 54, 461-475.

CROISILLE Y., JUNÉRA H., 1980. Recherche du lieu de synthèse de la vitellogénine chez le Crustacé Amphipode Orchestia gammarellus (Pallas). Démonstration, à l'aide d'anticorps spécifiques, de la présence de vitellogénine dans le tissu adipeux sous-épidermique des femelles en vitellogenèse secondaire. C. R. Acad. Sci. Paris, 290 D, 1487-1490.

DHAINAUT A., DE LEERSNYDER M., 1976. Etude cytochimique et ultrastructurale de l'évolution ovocytaire du crabe Eriocheir sinensis. I. Ovogenèse naturelle. Arch. Biol., 87, 261-282.

DURFORT M., 1977. Ultrastructura de la gonada femenina de Mytilicola intestinalis Steuer (Crustacea, Copepoda). Bol. R. Soc. esp. Hist. nat. (Biol.), 75, 71-86.

ERRIBABU D., SHYAMASUNDARI K., HANUMANTHA RAO K., 1978. The structure and cytochemistry of the oocytes in the crab Xantho bidentatus, Milne Edwards. Folia Histochem. Cytochem., 16. 57-64.

EURENIUS L., 1973. An electron microscope study of the developing oocytes of the crab Cancer pagurus L. with special reference to yolk formation (Crustacea). Z. Morph. Tiere, 75, 243254.

GANION L. R., KESSEL R. G., 1972. Intracellular synthesis, transport and packaging of proteinaceous yolk in oocytes of Orconectes immunis. J. Cell Biol., 52, 420-437. 
GARREAU DE LOUBRESSE N., 1974. Etude chronologique de la mise en place des enveloppes de l'œuf d'un Crustacé Phyllopode : Tanymastix lacunae. J. Microsc., 20, 21-38.

GOUDEAU M., LACHAISE F., 1980. "Endogenous yolk " as the precursor of a possible fertilization envelop in a crab (Carcinus maenas). Tiss. Cell., 12, 503-512.

HILTON I. F., 1932. The oogenesis of Calanus finmarchicus. Quart. J. microsc. Sci., 74, 193-222.

HINSCH G. W., CONE M. V., 1969. Ultrastructural observations of vitellogenesis in the spider crab, Libinia emarginata L. Cell Biol., 40, 336-342.

KESSEL R. G., 1968. Mechanism of protein yolk synthesis and deposition in Crustacean oocytes. Z Zelfforsch. Mikrosk. Anat., 89, 17-38.

LEDUC E. A., BERNHARD W., 1967. Recent modification of the glycol metacrylate embedding procedure. J. uttrastr. Res., 19, 196-199.

LOWE E., 1935. The anatomy of a marine Copepod, Calanus finmarchicus Gunnerus. Trans. R. Soc. Edinburgh, 58, 561-603.

MEUSY J. J., 1980. Vitellogenin, the extraovarian precursor of the protein yolk in Crustacea : a review. Reprod. Nutr. Develop., 20, 1-21.

PARK T. S., 1966. The biology of a Calanoid Copepod, Epilabidocera amphitrites Mc Murrish. Cellule, 66, 129-251.

PICAUD J. L., SOUTY C., 1980 a. Démonstration immunohistochimique de la présence de vitellogénine dans le tissu adipeux et dans l'hépatopancréas du Crustacé Isopode Oniscoìde Porcellio dilatatus (Brandt). C. R. Acad. Sci. Paris, 200 D. 123-125.

PICAUD J. L., SOUTY C., 1980 b. Démonstration par immunoautoradiographie de la synthèse de la vitellogénine par le tissu adipeux de Porcellio dilatatus (Brandt) (Crustacé, Isopodel. $C$. $R$. Acad. Sci. Paris, 290 D, $1019-1021$.

RAYMONT J. E. G., KRISHNASWAMY S., WOODHOUSE M. A., GRIFFIN R. L., 1974. Studies on the fine structure of Copedopa. Observations on Calanus finmarchicus (Gunnerus). Proc. roy. Soc. Edinburg, B 185, 409424.

RAZOULS S., 1973. Evolution des gonades chez les stades juvéniles d'un Copépode pélagique Temora stylifera (Calanoidea, Temoridael. C. R. Acad. Sci. Paris, 277 D, 1537-1539.

RAZOULS S., 1974. Maturité sexuelle et fécondité chez les femelles de Temora stylifera, Copépode pélagique (Copepoda, Calanoidea). Arch. Zool. exp. gen., 115, 387-399.

RAZOULS S., 1975. Fécondité, maturité sexuelle et différenciation de l'appareil génital des femelles de deux Copépodes planctoniques: Centropages typicus et Temora stylifera. Publ. Staz. zool. Napoli, 39 (suppl.), 297-306.

RAZOULS S., 1981. Etude écophysiologique de deux copépodes pélagiques. Essai d'application au mésoplancton des principes relatîs aux systèmes. Th. Doct. Etat, Univ. Paris VI.

REYNOLDS E. S., 1963. The use of lead citrate at high pH as an electron-opaque stain in electron microscopy. J. Cell Biol., 17, 208-212.

SABELLI SCANABISSI F., TRENTINI M., 1979. Ultrastructural observations on the oogenesis of Triops cancriformis (Crustacea, Notostraca). II. Early developmental stages of the oocyte. Cell. Tiss. Res., 201, 361-368.

SOUTY C., 1980. Bectron microscopic study of follicle cell development during vitellogenesis in the marine Crustacean Isopoda, Idotea balthica basteri. Reprod. Nutr. Dévelop., 20, $653-$ 663.

SOUTY C., PICAUD J. L., 1981. Vitellogenin synthesis in the fat body of the marine crustacean Isopoda, Idotea balthica basteri, during vitellogenesis. Reprod. Nutr. Dévelop., 21, 95-101.

THIÉRY J. P., RAMBOURG A., 1974. Cytochimie des polysaccharides. J. Microsc., 21. 225-232.

TRENTINI M., SABELU SCANABISSI F., 1978. Ultrastructural observations on the oogenesis of Triops cancriformis (Crustacea, Notostraca). I. Origin and differentiation of nurse cells. Cell Tiss. Res., 194. 71-77.

VARADARAJAN S., SUBRAMONIAM T., 1980. Histochemical investigations on vitellogenesis of an anomouran crab, Clibanarius clibanarius. Int. J. Invert. Reprod., 2, 47-58.

WOLIN E. M., LAUFER H., ALBERTINI D. F., 1973. Uptake of the yolk protein lipovitellin by developing crustacean oocytes. Develop. Biol., 35, 160-170.

ZERBIB C., 1973. Contribution à l'étude ultrastructurale de l'ovocyte chez le Crustacé Amphipode Orchestia gammarella Pallas. C.R. Acad. Sci. Paris, 277 D, 1209-1212. 
ZERBIB C., 1975. Première observation de granules corticaux dans l'ovocyte d'un Crustacé, l'Amphipode Orchestia gammarellus (Pallas). C.R. Acad. Sci. Paris, 281 D, 1345-1347.

ZERBIB C., 1976. Nature chimique des enclaves vitellines de l'ovocyte du Curstacé Amphipode Orchestia gammare/lus (Pallas). Ann. Histochem., 21, 279-295.

ZERBIB C., 1978. Ovogenèse chez le Crustacé Amphipode Orchestia gammarellus (Pallas) : contribution à son étude cytologique et physiologique. Th. Doct. Etat, Univ. Paris VI.

ZERBIB C., 1979. Etude ultrastructurale de l'ovocyte en vitellogenèse chez les Ecrevisses Astacus astacus et $A$. leptodacty/us. Int. J. Invert. Reprod., 1, 289-295.

ZERBIB C., 1980. Ultrastructural observation of oogenesis in the Crustacea Amphipoda Orchestia gammarellus (Pallas). Tiss. Cell, 12, 47-62. 